Journal of Bangladesh Academy of Sciences, Vol. 34, No. 2, 103-114, 2010

\title{
ON A SIMPLE METHOD OF DETERMINING THE HOMOLOGY AND THE COHOMOLOGY OF FINITELY PRESENTED GROUPS
}

\author{
SUBRATA MAJUMDAR ${ }^{1}$ AND NASIMA AKHTER \\ Department of Mathematics, Rajshahi University, Rajshahi-6205, Bangladesh
}

\begin{abstract}
In this paper the authors obtained a method of constructing free resolutions of $Z$ for finitely presented groups directly from their presentations by extending Lyndon's 3-term partial resolution to a full-length resolution. Authors resolutions and the method of their construction are such that free generators of the modules and the boundary homomorphisms are directly and explicitly obtained by solving of linear equations over the corresponding integral group rings, and hence these are immediately applicable for computing homology and cohomology of the groups for arbitrary coefficient modules. Authors have also described a general situation where their method is valid. The method has been used for a number of classes of group including Fuchsian groups, a few Euclidean crystallographic groups, NEC groups, the fundamental groups of a few interesting manifolds, groups of isometries of the hyperbolic plane and a few nilpotent groups of class 2 .
\end{abstract}

Key words: Group presentation, Free resolution, Homology, Cohomology

\section{INTRODUCTION}

One of the ways of determining the homology and cohomology of groups with arbitrary coefficient modules is to construct a suitable projection ZG-resolution of Z. The standard resolution, the bar resolution and the Gruenberg resolution are very well-known, but these are more useful for development of theory than for computation of homology for specific groups.

For computational purpose the free resolution of Eilenberg and MacLane (1954) for cyclic groups had been the starting point. This was generalised on one hand for singlerelator group by Lyndon (1950) using a vital group theoretic tool 'the identity theorem' proved by himself. His ideas were further developed by Swan (1969) and Gildenhuys (1976). Using the solution of the identity Huebschmann (1993) obtained the cohomology of aspherical groups. Another approch on generalisation from cyclic groups was to construct free resolutions for finitely generated abelian groups i.e., groups which are finite direct products of cyclic groups. This approach uses that fact that $Z\left(G_{1} \times G_{2}\right) \cong Z\left(G_{1}\right) \otimes_{Z} Z\left(G_{2}\right)$ and utilises the Kunneth Formula for the tensor product of complexes. Huebschmann (1989) generalised this method to obtain the cohomology of the class of nilpotent groups of class 2 by developing a perturbation technique which, in essence, perturbs the tensor product structure in $M(Q) \otimes M(N)$ for an extension.

\footnotetext{
${ }^{1}$ Corresponding author: <prof.subrata.majumdar@gmail.com>.
} 
$E: 1 \rightarrow N \rightarrow G \rightarrow G / N=Q \rightarrow 1 \quad(N, Q$ non-abelian). Here $M(N)$ and $M(Q)$ are algebra models for the unreduced bar construction. $M(Q) \otimes Z G$ is an augmented differential graded commutative algebra. $G$ may be regarded as the direct product $Q \times N$ with a perturbation, and a free ZG-resolution is constructed by perturbing the tensor product structure in $M(Q) \otimes M(N)$. The basic underlying idea was latent in Wall (1961).

If a group $\mathrm{G}$ is given an extension $1 \rightarrow N \rightarrow G \rightarrow G / N \rightarrow 1$, the relationship of the homology and cohomology of $G$ with those of $N$ and $G / N$ is provided by Lyndon's work (1950) using spectral sequences - a sophisticated machinery of homological algebra. A corresponding result for fiber spaces is due to Leray-Serre, theorem (MacLane 1963).

A recent method of construction of resolutions uses a technique called 'a rewriting system'. It was developed and applied for monoids by Anick (1986) and Squire (1987) for extensions of groups by Groves (1989) and Carbone (1996).

In this paper authors shall describe an entirely new method of constructing a free ZG-resolution of $Z$ for groups $G$ from their presentations. Lyndon's work (1950) contains the essential ingredients.

Authors shall also describe a general situation where this method is valid. Through a series of papers of Majumdar and Akhter $(2002,2003,2008)$ the method has been applied to many classes of groups including the class of non Euclidean groups with nonorientable quotient spaces and no reflexions, a metacyclic groups, a factor group of the Heisenberg group, an infinite group related to Fibonacci groups, the trefoil kont group, the fundamental group of the geometrical limit of a sequence of quasi-fuchsian manifolds, a few groups of isometry of the hyperbolic plane. Previously, such method was tacitly applied by Majumdar $(1970,1971,1983,1984,1987)$ to compute the homology and the cohomolgy of a few classes of groups including the fuchsian groups and a number of Euclidean crystallographic groups.

The importance and the significance of the method lies in its simplicity of construction and its ability to yield the homology and the cohomology groups without any simplification or reduction of the resolution. No sophisticated or elaborate homological or group-theoretic tools are needed. The technique lies in extending Lyndon's partial resolution to a full-length resolution through step-by-step solutions of system of linear equations over the integral group ring. The consideration whether the group is given as an extension, or it is abelian or almost abelian, such as nilpotent of class 2 , or it satisfies small cancellation properties, are not at all important. The method works so long as the systems of equations are soluble with the help of the relations. For groups whose homology and cohomology have been already determined, this method appears to provide an easier way of computation. 


\section{Lyndon's Partial Resolution}

Lyndon obtained a partial resolution for a group from its presentation. The authors describe it below.

Theorem 2.1 [(1950), (1965), (1960)]

Let $G=F / R$, where $F$ is the free group freely generated by $x_{1}, \ldots, x_{m}$ and $R$ is the normal subgroup generated by $r_{1}, \ldots, r_{n}$ in $F$. Then there is a ZG-free partial resolution of $Z$ given by

(A) $\quad Y_{1} \stackrel{d_{1}}{\longrightarrow} Y_{0} \stackrel{d_{0}}{\longrightarrow} Z G \stackrel{\varepsilon}{\longrightarrow} Z \rightarrow 0$

where $Y_{0}, Y_{1}$ are ZG-modules free on $\left\{\alpha_{1}, \ldots, \alpha_{m}\right\}$ and $\left\{\beta_{1}, \ldots, \beta_{n}\right\}$ respectively, and $\varepsilon, d_{0}, d_{1}$ are $Z \mathrm{G}$-homomorphisms given by

$\varepsilon(g)=1$, for all $g \in G, d_{0}\left(\alpha_{i}\right)=\pi\left(x_{i}-1\right)$, for each $i$, and

$$
d_{1}\left(\beta_{j}\right)=\sum_{i=1}^{m} \alpha_{i} \pi\left(\frac{\partial r_{j}}{\partial x_{i}}\right), \text { for each } j
$$

\section{Method of Extension of $\left(A_{1}\right)$ to a Full Resolution}

The authors shall develop a method of extending $\left(A_{1}\right)$ to a full free ZG-resolution $(A)$ of Z:

$\ldots \rightarrow Y_{q+1} \stackrel{d_{q+1}}{\longrightarrow} Y_{q} \stackrel{d_{q}}{\longrightarrow} \ldots \rightarrow Y_{2} \stackrel{d_{2}}{\longrightarrow} Y_{1} \stackrel{d_{1}}{\longrightarrow} Y_{0} \stackrel{d_{0}}{\longrightarrow} Z G \stackrel{\varepsilon}{\longrightarrow} Z \rightarrow 0$ To

extend $\left(A_{1}\right)$ authors first find a free ZG-module $Y_{2}$ and ZG-homomorphism $d_{2}: Y_{2} \rightarrow Y_{1}$ such that

$\operatorname{Ker} d_{1}=\operatorname{Im} d_{2}$. An element $\sum_{j=1}^{n} \beta_{j} \gamma_{j}$ belongs to $\operatorname{Ker} d_{1}$ if and only if

$$
d_{1}\left(\sum_{j=1}^{n} \beta_{j} \gamma_{j}\right)=0
$$

i.e., if and only if $\sum_{j=1}^{n} \pi\left(\frac{\partial r_{j}}{\partial x_{i}}\right) \gamma_{j}=0$, for each $i$, since $Y_{0}$ is free on $\alpha_{i}{ }^{\prime} s$. The kernel of $d_{1}$ will be known if the authors know all the $\gamma_{j}{ }^{\prime} s$ satisfying the system of linear equations.

(1) $\ldots \sum_{j=1}^{n} \pi\left(\frac{\partial r_{j}}{\partial x_{i}}\right) \gamma_{j}=0$, for $i=1,2, \ldots, m$.

If authors can solve the system of linear equations (1) and obtain the solutions of the systems in the form

(1') $\ldots . . \quad \gamma_{j}=\sum_{k=1}^{u} \lambda_{j k} \gamma_{k}^{\prime}$ 
where $\lambda_{j k}, \gamma^{\prime} \in Z G$ and each $\lambda_{j k}$ is known and $\gamma_{k}^{\prime}$ are arbitrary, then $\operatorname{Imd}_{2}$ is the right ZGmodule consisting of all

$$
\gamma=\sum_{j=1}^{n} \beta_{j}\left(\sum_{k=1}^{u} \lambda_{j k} \gamma_{k}^{\prime}\right)=\sum_{k=1}^{u} \sum_{j=1}^{n}\left(\beta_{j} \lambda_{j k}\right) \gamma_{k}^{\prime}
$$

where $\lambda_{j k}$ 's are given by (1') and $\gamma_{k}^{\prime}$ are arbitrary. This suggests that we take $Y_{2}$ to be the right ZG-module free on, say, $\left\{\delta_{1}, \ldots, \delta_{u}\right\}$ in 1-1 correspondence with $\left\{\gamma_{1}^{\prime}, \ldots, \gamma_{u}^{\prime}\right\}$, and define the ZG-homomorphism $d_{2}: Y_{2} \rightarrow Y_{1}$ by

$$
d_{2}\left(\delta_{k}\right)=\sum_{j=1}^{n} \beta_{j} \lambda_{j k}
$$

These definitions ensure that the kernel of $d_{1}$ is contained in the image of $d_{2}$.

The reverse inclusion follows from the following:

For each $\sum_{k=1}^{u} \delta_{k} \gamma_{k}^{\prime}$ in $Y_{2}$

$$
\begin{aligned}
d_{1} d_{2}\left(\sum_{k=1}^{u} \delta_{k} \gamma_{k}^{\prime}\right) & =d_{1}\left(\sum_{j=1}^{n} \beta_{j}\left(\sum_{k=1}^{u} \lambda_{j k} \gamma_{k}^{\prime}\right)\right) \\
& =\sum_{j=1}^{n}\left(\sum_{i=1}^{m} \alpha_{i} \pi\left(\frac{\partial r_{j}}{\partial x_{i}}\right)\right)\left(\sum_{k=1}^{u} \lambda_{j k} \gamma_{k}^{\prime}\right) \\
& =\sum_{i=1}^{m} \alpha_{i}\left(\sum_{j=1}^{n} \pi\left(\frac{\partial r_{j}}{\partial x_{i}}\right) \sum_{k=1}^{u} \lambda_{j k}\right) \gamma_{k}^{\prime}=0,
\end{aligned}
$$

since $\sum_{k=1}^{u} \lambda_{j k} \gamma_{k}^{\prime}$ is a solution of $\sum_{j=1}^{n} \pi\left(\frac{\partial r_{j}}{\partial x_{i}}\right) \gamma_{j}=0$

In this way, the solution of the system of equations (1) leads to extension of the partial resolution $\left(A_{1}\right)$ by one more term $Y_{2}$ to yield

$\left(\mathrm{A}_{2}\right) \ldots$

$$
Y_{2} \stackrel{d_{2}}{\longrightarrow} Y_{1} \stackrel{d_{1}}{\longrightarrow} Y_{0} \stackrel{d_{0}}{\longrightarrow} Z G \stackrel{\mathcal{E}}{\longrightarrow} Z \rightarrow 0
$$

To extend $\left(A_{2}\right)$ by one more term we turn our attention to Ker $d_{2}$. We proceed almost similarly.

$$
\text { Let } \sum_{k=1}^{u} \delta_{k} \gamma_{k} \in \operatorname{Ker} d_{2},
$$

then $\sum_{k=1}^{u} \lambda_{j k} \gamma_{k}=0$ since $Y_{1}$ is free on $\beta_{j}$ 's

for each $j=1, \ldots, n$.

As before, to find the kernel of $d_{2}$ authors solve the system of linear equations. 
(2) $\ldots \quad \sum_{k=1}^{u} \lambda_{j k} \gamma_{k}=0, \quad(j=1, \ldots, n)$

If authors can get the solutions of the system of linear equations (2) and the solutions are in the form

$\left(2^{\prime}\right) \ldots \quad \gamma_{k}=\sum_{j=1}^{v} \mu_{k l} \gamma_{l}^{\prime}$

where $\mu_{k l}, \gamma_{l}^{\prime} \in Z G$ and $\mu_{k l}$ are known and $\gamma_{l}^{\prime}$ are arbitrary, then authors obtain the next term $Y_{3}$ and the next homomorphism $\mathrm{d}_{3}$, so that the corresponding extension of $\left(A_{2}\right)$ is exact at $Y_{2}$, by defining $Y_{3}$ as a right ZG-module free on $\left\{\rho_{1}, \ldots, \rho_{v}\right\}$ in $1-1$ correspondence with $\left\{\gamma_{1}^{\prime}, \ldots, \gamma_{v}^{\prime}\right\}$ and defining the ZG homomorphism $d_{3}: Y_{3} \rightarrow Y_{2}$ by

$$
d_{3}\left(\rho_{1}\right)=\sum_{k=1}^{u} \delta_{k} \mu_{k l}
$$

As before, it may be verified that the sequence $\left(A_{2}\right)$ has been extended by the term $Y_{3}$ to yield the exact sequence

$\left(A_{3}\right) \ldots$

$$
Y_{3} \stackrel{d_{3}}{\longrightarrow} Y_{2} \stackrel{d_{2}}{\longrightarrow} Y_{1} \stackrel{d_{1}}{\longrightarrow} Y_{0} \stackrel{d_{0}}{\longrightarrow} Z G \stackrel{\varepsilon}{\longrightarrow} Z \rightarrow 0
$$

Authors now suppose that $\left(A_{3}\right)$ has been extended to an exact sequence of right ZGmodules and ZG-homomorphisms

$\left(A_{s}\right)$

$$
Y_{s} \stackrel{d_{s}}{\longrightarrow} Y_{s-1} \stackrel{d_{s-1}}{\longrightarrow} \ldots Y_{1} \stackrel{d_{1}}{\longrightarrow} Y_{0} \stackrel{d_{0}}{\longrightarrow} Z G \stackrel{\varepsilon}{\longrightarrow} Z \rightarrow 0
$$

$s>3$, where $Y_{s}$ and $Y_{s-1}$ are free on $\left\{\alpha_{1}^{s}, \ldots, \alpha_{m_{s}}^{s}\right\}$ and $\left\{\alpha_{1}^{s-1}, \ldots, \alpha_{m_{s-1}}^{s-1}\right\}$, respectively and $d_{s}$ is defined by $d_{s}\left(\alpha_{i}^{s}\right)=\sum_{j=1}^{m_{s-1}} \alpha_{j}^{s-1} \lambda_{j i}^{s}$,

the $\alpha_{i}^{s}, \alpha_{j}^{s-1}$ and $d_{\mathrm{s}}$ having been obtained as in $\left(A_{2}\right)$ and $\left(A_{3}\right)$.

From here, if we consider Kre $d_{s}$ and fine out the solutions of the system of linear equations $\sum_{i=1}^{m_{s}} \lambda_{j i}^{s} \gamma_{i}^{s}=0, j=1, \ldots, m_{(s-1)}$ in $\gamma_{i}^{s}$ over $\mathrm{ZG}$, and if solutions are of the form $\gamma_{i}^{s}=\sum_{j=1}^{m_{s+1}} \lambda_{i j}^{s+1} \gamma_{j}^{s+1}$ with $\lambda_{i j}^{s+1}$ known and $\gamma_{j}^{s+1}$ arbitrary elements of ZG, then we define $Y_{s+1}$ as a right ZG-module free on $\left\{\alpha_{1}^{s+1}, \ldots, \alpha_{m_{s+1}}^{s+1}\right\}$ in $1-1$ correspondence with $\left\{\gamma_{1}^{s+1}, \ldots, \gamma_{m_{s+1}}^{s+1}\right\}$ and define ZG-homomorphism $d_{s+1}: Y_{s+1} \rightarrow Y_{s}$ by $d_{s+1}\left(\alpha_{i}^{s+1}\right)=\sum_{j=1}^{m_{s}} \alpha_{j}^{s} \lambda_{j i}^{s+1}$.

Then Im $d_{s+1}=\operatorname{Ker} d_{s}$ and authors obtain an exact sequence of ZG-modules and ZGhomomorphisms

$$
\left(A_{s+1}\right) \ldots \quad Y_{s+1} \stackrel{d_{s+1}}{\longrightarrow} Y_{s} \stackrel{d_{s}}{\longrightarrow} \ldots Y_{1} \stackrel{d_{1}}{\longrightarrow} Y_{0} \stackrel{d_{0}}{\longrightarrow} Z G \stackrel{\varepsilon}{\longrightarrow} Z \rightarrow 0
$$


which is an extension of $\left(A_{S}\right)$.

Thus authors obtain a full free ZG-resolution of $Z$.

Authors have thus proved

\section{Theorem 2.2}

Let $G=\langle X ; Y\rangle$ be a free presentation of $G$, where $X=\left\{x_{1}, \ldots x_{m}\right\}, Y=\left\{r_{1}, \ldots r_{n}\right\}$. Let $F$ be a free group freely generated by $\left\{x_{1}, \ldots x_{m}\right\}$ and $R$ the normal subgroup generated by $\left\{r_{1}, \ldots r_{n}\right\}$ in $F$. Then the following is a free ZG-resolution of $Z$ :

(A) $\ldots \quad \rightarrow Y_{q+1} \stackrel{d_{q+1}}{\longrightarrow} Y_{q} \stackrel{d_{q}}{\longrightarrow} \ldots Y_{1} \stackrel{d_{1}}{\longrightarrow} Y_{0} \stackrel{d_{0}}{\longrightarrow} Z G \stackrel{\varepsilon}{\longrightarrow} Z \rightarrow 0$

Here, $Y_{0}$ is a right ZG-module free on $\left\{\alpha_{1}^{0}, \ldots, \alpha_{m_{0}}^{0}\right\}$, where $m_{0}=m$

$Y_{1}$ is a right ZG-module free on $\left\{\alpha_{1}^{1}, \ldots, \alpha_{m_{1}}^{1}\right\}$, where $m_{1}=n$

$Y_{2}$ is a right ZG-module free on $\left\{\alpha_{1}^{2}, \ldots, \alpha_{m_{2}}^{2}\right\}$,

where $m_{2}$ is the number of $\gamma_{j}^{2}$ 's, the latter being given by the solutions $\sum_{j=1}^{m_{2}} \lambda_{i j}^{2} \gamma_{j}^{2}$ of the system of linear equations $\sum_{i=1}^{m_{1}} \lambda_{i j}^{1} \gamma_{i}^{1}=0$ in $\gamma_{i}^{1}$ 's and the ZG-homomorphisms $\varepsilon, d_{0}, d_{1}$ and $d_{2}$ are given by $\varepsilon(g)=1$ for all $g \in G, d_{0}\left(\alpha_{i}^{0}\right)=\pi\left(x_{i}-1\right), i=1, . ., m$, where $\pi$ is the ring homomorphism $\mathrm{ZF} \rightarrow \mathrm{ZG}$ induced by the canonical homomorphism $F \rightarrow F / R=G$.

$d_{1}\left(\alpha_{j}^{1}\right)=\sum_{i=1}^{m} \alpha_{i}^{0} \pi\left(\partial r_{j} / \partial x_{i}\right)=\sum_{i=1}^{m} \alpha_{i} \lambda_{i j}^{1}$, writing $\lambda_{i j}^{1}=\pi\left(\partial r_{j} / \partial x_{i}\right)$
$d_{1}\left(\alpha_{j}^{2}\right)=\sum_{i=1}^{m_{1}} \alpha_{i}^{1} \lambda_{i j}^{2}, \lambda_{i j}^{2}$ 's are defined by the solution $\sum_{j=1}^{m_{2}} \lambda_{i j}^{2} \gamma_{j}^{2}$ of the system of linear equations $\sum_{i=1}^{m_{1}} \lambda_{i j}^{1}=0$ in $\gamma_{i}^{1}$ 's. We assume $d_{3}, \ldots, d_{q}$ and $Y_{3}, \ldots, Y_{q}$ have been defined as follows:

$Y_{u}$ is right ZG- module free on $\left\{\alpha_{1}^{u}, \ldots \alpha_{m_{u}}^{u}\right\}, 3 \leq u \leq q$, and $d_{u:} Y_{u} \rightarrow Y_{u-1}$ is a ZGhomomorphism given by $d_{u}\left(\alpha_{i}^{u}\right)=\sum_{i=1}^{m_{u-1}} \alpha_{i}^{u-1} \lambda_{i j}^{u}$. Then $Y_{q+1}$ and $d_{q+1}$ are defined as follows.

$Y_{q+1}$ is a right ZG-module free on $\left\{\alpha_{1}^{q+1}, \ldots, \alpha_{m_{q+1}}^{q+1}\right\}$, where $m_{q+1}$ is the number of $\gamma_{j}^{q+1}$, $\mathrm{s}$ the latter being defined by the solutions $\sum_{j=1}^{m_{q+1}} \lambda_{i j}^{q+1} \gamma_{j}^{q+1}$ of the system of equations $\sum_{i=1}^{m_{q}} \lambda_{i j}^{q} \gamma_{i}^{q}=0$ in $\gamma_{i}^{q}$, s and $d_{q+1}: \quad Y_{q+1} \rightarrow Y_{q}$ is a ZG-homomorphism given by $d_{q+1}\left(\alpha_{j}^{q+1}\right)=\sum_{i=1}^{m_{q}} \alpha_{i}^{q} \lambda_{i j}^{q+1}$. 


\section{Remark}

Authors see that the ZG-homomorphism $d_{q}: Y_{q} \rightarrow Y_{q+1}$ for $q \geq 2$ in $(A)$ is given by

$$
\begin{aligned}
d_{q}\left(\sum_{j=1}^{m_{q}} \alpha_{j}^{q} \gamma_{j}\right) & =\sum_{j=1}^{m_{q}}\left(\sum_{i=1}^{m_{q-1}} \alpha_{i}^{q-1} \lambda_{i j}^{q}\right) \gamma_{j} \\
& =\sum_{i=1}^{m_{q-1}} \alpha_{i}^{q-1}\left(\sum_{j=1}^{m_{q}} \lambda_{i j}^{q} \gamma_{j}\right) \text { for all } \sum_{j=1}^{m_{q}} \alpha_{j}^{q} \gamma_{j} \in Y_{q} .
\end{aligned}
$$

If we write, $\sum_{j=1}^{m_{q}} \alpha_{j}^{q} \gamma_{j}$ as $\left(\gamma_{1}, \ldots, \gamma_{m_{q}}\right)$, then

$$
\begin{aligned}
d_{q}\left(\gamma_{1}, \ldots, \gamma_{m_{q}}\right) & =\left(\sum_{j=1}^{m_{q}} \lambda_{1 j}^{q} \gamma_{j}, \ldots, \sum_{j=1}^{m_{q}} \lambda_{m_{q-1} j}^{q} \gamma_{j}\right) \\
& =\left(\lambda_{11}^{q} \gamma_{1}+\ldots+\lambda_{1 m_{q}}^{q} \gamma_{m_{q}}, \ldots, \lambda_{m_{q-1}, 1}^{q} \gamma_{1}+\ldots+\lambda_{m_{q-1}, m_{q}}^{q} \gamma_{m_{q}}\right) .
\end{aligned}
$$

\section{Determination of Homology and Cohomology}

For any left ZG-module A the homology group $H_{n}(G, A)$ are giver by the homology of the complex of abelian groups:

$(\mathrm{P}) \ldots \rightarrow Y_{q+1} \otimes A \stackrel{d_{q} \otimes 1_{A}}{\longrightarrow} Y_{q} \otimes A \rightarrow \ldots Y_{1} \otimes A \stackrel{d_{1} \otimes 1_{A}}{\longrightarrow} Y_{0} \otimes A \stackrel{d_{0} \otimes 1_{A}}{\longrightarrow} Z G \otimes A \rightarrow 0$

The isomorphisms $R \underset{R}{\otimes} B \cong B$ and $\sum_{a} A_{a} \underset{R}{\otimes} B \cong \sum_{a}\left(A_{a} \underset{R}{\otimes} B\right)$ imply that the homology of $(\mathrm{P})$ is isomorphic to the homology of the complex:

$\left(\mathrm{P}^{\prime}\right) \ldots \rightarrow A^{m_{q+1}} \stackrel{\bar{d}_{q+1}}{\longrightarrow} A^{m_{q}} \rightarrow \ldots \rightarrow A^{m_{2}} \stackrel{\bar{d}_{2}}{\longrightarrow} A^{n} \stackrel{\bar{d}_{1}}{\longrightarrow} A^{m} \stackrel{\bar{d}_{0}}{\longrightarrow} A \rightarrow 0$

in each dimension, where $A^{q}$ denotes the direct sum of $q$ copies of $A \bar{d}_{q}$ are induced by $d_{q} \otimes 1_{A}$ and are given by

$(\overline{\mathrm{P}}) \ldots \quad \bar{d}_{q}\left(a_{1}, \ldots, a_{m_{q}}\right)=\left(\lambda_{11}^{q} a_{1}+\ldots+\lambda_{1 m_{q}}^{q} a_{m_{q}}, \ldots, \lambda_{m_{q-1}, 1} a_{1}+\ldots+\lambda_{m_{q-1}, m_{q}} a_{m_{q}}\right), q=1,2,3, \ldots$

Then the $q$-th homology group of $G$ with coefficient in $A$ is

$\left(\mathrm{P}^{\prime \prime}\right) \ldots \quad H_{q}(G, A)=\operatorname{Ker} \bar{d}_{q-1} / \operatorname{Im} \bar{d}_{q}, q \geq 1, H_{0}(G, A)=A / \operatorname{Im} \bar{d}_{0}$

For any right ZG-module $A$, the cohomology group $H^{n}(G, A)$ are given by the homology of the complex:

$\operatorname{Hom}\left(Y_{q}, A\right) \stackrel{d_{q}^{*}}{\longleftarrow} \ldots \leftarrow \operatorname{Hom}\left(Y_{1}, A\right) \stackrel{d_{1}^{*}}{\longleftarrow} \operatorname{Hom}\left(Y_{0}, A\right) \stackrel{d_{0}^{*}}{\longleftarrow} \operatorname{Hom}(Z G, A) \leftarrow 0$

where $d_{q}^{*}=\operatorname{Hom}\left(d_{q}, 1_{A}\right)$.

The natural isomorphisms $\operatorname{Hom}_{\mathrm{R}}(R, B) \cong B$ and 


$$
\operatorname{Hom}_{R}\left(\sum_{\alpha=1}^{l} B_{\alpha}, B\right) \cong \sum_{\alpha=1}^{l} \operatorname{Hom}_{R}\left(B_{\alpha}, B\right)
$$

so that the homology of the above complex is isomorphic to the complex:

(Q) $\ldots \ldots \stackrel{d_{q+1}^{* *}}{\longleftarrow} A^{m_{q}} \stackrel{d_{q}^{* *}}{\longleftarrow} \ldots \stackrel{d_{3}^{* *}}{\longleftarrow} A^{m_{2}} \stackrel{d_{2}^{* *}}{\longleftarrow} A^{n} \stackrel{d_{1}^{* *}}{\longleftarrow} A^{m} \stackrel{d_{0}^{* *}}{\longleftarrow} A \leftarrow 0$

in each dimension, where $A^{q}$ is the direct sum of $q$ copies of $A$ and $d_{i}^{* *}$ are induced by $d_{i}^{*}$ and are given by

$$
\begin{aligned}
& \left(\mathrm{Q}^{*}\right) \ldots d_{q}^{* *}\left(a_{1}, \ldots, a_{m_{q-1}}\right) \\
& =\left(\lambda_{11}^{q} a_{1}+\lambda_{21}^{q} a_{2}+\ldots+\lambda_{m_{q-1,1}}^{q} a_{m_{q-1}}, \ldots, \lambda_{1, m_{q}}^{q} a_{1}+\lambda_{2, m_{q}}^{q} a_{2}+\ldots+\lambda_{m_{q-1}, m_{q}} a_{m_{q-1}}\right) \\
& \mathrm{q}=1,2,3, \ldots .
\end{aligned}
$$

Then the $q$-th cohomology group of $G$ with coefficients in $A$,

$$
\left(\mathrm{Q}^{* *}\right) \ldots \quad H^{q}(G, A) \cong \frac{\operatorname{Ker}_{q}^{* *}}{\operatorname{Imd}_{q-1}^{* *}}, q \geq 1, H^{0}(G, A) \cong \operatorname{Kerd}_{0}^{* *}
$$

Thus the knowledge of the $\lambda_{i j}^{q}$ 's enables us to know $H_{q}(G, A)$ and $H^{q}(G, A)$ immediately from $(\overline{\mathrm{P}})$ and $\left(\mathrm{P}^{\prime \prime}\right)$ and $\left(\mathrm{Q}^{*}\right)$ and $\left(\mathrm{Q}^{* *}\right)$, respectively.

\section{Integral Homology Groups of $G$}

The expression of the above homology groups and cohomology groups can be simplified when $A$ is abelien group regarded as a trivial ZG-module. By the Universal coefficient theorems for homology of groups and for cohomology of groups (Majumdar 1970), it is sufficient to determined the homology groups $H_{n}(G, Z)$ and to determine the cohomology groups $H^{n}(G, Z)$, where $\mathrm{Z}$ is regarded as a trivial ZG-module.

The effectiveness of this method depends on the possibility and the ability of solving the systems of the linear equations arising at different steps. Sometimes it may not be possible to solve the system at each step. In the possible situations the resolutions is nice in the sense that each dimension the homology and the cohomology of the group can be obtained explicitly by a finite process. It is possible that we may have a trivial solution after finite number of steps. Also it is possible that, after a finite number of steps, the solution repeat with a finite period of step- numbers. In the first situation the resolution is finite and the group has a finite homological dimension. In the latter case, the homology becomes periodic after a finite number of steps. In these cases the resolutions are nicer in the sense that a single finite process displays explicitly the homology and the cohomology in all dimensions. If impossibility appears after a finite number of steps, we obtain an effective partial resolution which immediately yields the lower dimensional homology and the cohomology. 
Authors shall now describe a general situation in which the full resolution $(A)$ exists and is obtainable.

For this authors start with the following definition.

\section{Definition 2.1}

Let $R$ be a ring with 1 and $A=\left(a_{i j}\right)$ an $n \times n^{\prime} \quad\left(n \leq n^{\prime}\right)$ matrix over $R$ with the properties

(i) $a_{i j}=0, i \neq j, i=1, \ldots, r,(r \leq n)$

(ii) $a_{r+k, j}=0, j \neq k$ or $j \neq r+k, k=1, \ldots, s \leq r$

(iii) $a_{r+s+m, j}=0, m=1, \ldots, u, u=\frac{1}{2}(n-r-s)$

(iv) $a_{r+s+u+m, j}=0, j \neq r+s+m$ or $j \neq r+s+u+m$.

(v) $a_{i, j}=0, j \geq n$.

(vi) $A n n_{R} a_{i i}=b_{i i} R, A n n_{L b_{i i}}=R a_{i i} \quad b_{i i}$ and $a_{r+i, i}$ commute with each other and $a_{r+k, r+k}=b_{k k}$, for each $i$ and $k$.

(vii) $a_{r+s+u+m, r+s+m}$ has a left annihilator $R c_{r+s+u+m, r+s+m}$ such that $a_{r+s+u+m, r+s+m}$ and $c_{r+s+u+m, r+s+m}$ commute with $a_{r+s+u+m, r+s+u+m}$, the latter has trivial right annihilator and $A n n_{R} c_{r+s+u+m, r+s+m}=a_{r+s+u+m, r+s+m} R$.

Such a matrix will be called a standard matrix and the form of the matrix will be the standard form.

Now, if $A=\left(a_{i j}\right)$ is an $n \times n^{\prime}\left(n \leq n^{\prime}\right)$ standard matrix over $R$, Then the system of linear equations

$$
\begin{aligned}
& A X=0 \text {, where } X=\left(\begin{array}{c}
x_{1} \\
\vdots \\
x_{n}
\end{array}\right), 0=\left(\begin{array}{c}
0 \\
\vdots \\
0
\end{array}\right) \text { is given by the following: } \\
& \text { (I) } a_{11} x_{1}=0, \ldots, a_{r r} x_{r}=0 \\
& \text { (II) } a_{r+1,1} x_{1}+a_{r+1, r+1} x_{r+1}=0 \\
& \quad \ldots \quad \ldots \\
& \quad a_{r+s, s} x_{s}+a_{r+s, r+s} x_{r+s}=0 \\
& \text { (III) } a_{r+s+u+1, r+s+1} x_{r+s+1}+a_{r+s+u+1, r+s+u+1} x_{r+s+u+1}=0 \\
& \quad \ldots \quad \ldots \\
& \quad a_{r+s+2 u, r+s+1} x_{r+s+u}+a_{r+s+2 u, r+s+2 u} x_{r+s+2 u}=0
\end{aligned}
$$


The solution of the above system of equations are given by

(IV) $x_{1}=b_{11} \alpha_{1}, \ldots, x_{r}=b_{r r} \alpha_{r}$, for some $\alpha_{1}, \ldots, \alpha_{r} \in R$, where $b_{i i} R$ is the right annihilator of $a_{i i}$. Also,

(V) $x_{r+k}=-a_{r+k, k} \alpha_{k}+a_{k k} \alpha_{r+k}, k=1, \ldots, s$ for some $\alpha_{k} \alpha_{r+k} \in R$.

(VI) $x_{r+s+u+m}=a_{r+s+u+m, r+s+u+m} \alpha_{r+s+u+m}, m=1, \ldots, u$, for some $\alpha_{r+s+u+m} \in R$.

(VII) $x_{r+s+m}=c_{r+s+u+m, r+s+m} \alpha_{r+s+m}-a_{r+s+u+m, r+s+u+m} \alpha_{r+s+u+m}$ for some $\alpha_{r+s+m} \in R$.

(VIII) $x_{j}, j>r+s+2 u$, are free variables.

The following operations

(i) $R_{i} \leftrightarrow R_{j}$; (ii) $R_{i} \rightarrow c R_{i}$ (c is a unit in $R$ ); (iii) $R_{i} \rightarrow R_{i}+c R_{j}$ on the rows of $A=\left(a_{i j}\right)$ are elementary row operations.

A matrix $A$ is equivalent to a matrix $B$, written $A \sim B$ if there exists a finite sequence of elementary row operations transforming $A$ into $B$. If $A \sim B, A X=0$ and $B X=0$ have the same solution-sets.

A matrix $A$ over $R$ is called perfect if there exits a sequence $\left\{A_{n}\right\}$ of standard matrices over $R$ such that

(i) $A_{1} \sim A$,

(ii) For each $n \geq 1, A_{n+1} \sim B_{n}$, where $B_{n}$ is the matrix determined by the solutions of the system of linear equations $A_{n} X=0$, i.e., if the solutions of $A_{n} X=0$ are given by $x_{i}=\sum_{j=1}^{v} b_{i j} y_{j}, i=1, \ldots, u$.

Then $B_{n}=\left(b_{i j}\right)$

$\left\{A_{n}\right\}$ is called the sequence of standard matrices associated with $A$, and for each $n, B_{n}$ is called associate of $A_{n}$.

The above discussion proves the validity of the following Theorem:

\section{Theorem 2.3}

Let $G$ be a group as in Theorem 2.2 and such that $A=\left(\pi\left(\partial r_{j} / \partial x_{i}\right)\right)$ is a perfect matrix with an associated sequence of standard matrices $\left\{A_{n}\right\}$. Then the following is a free ZG-resolution of $Z$

$$
\ldots \stackrel{d_{q+1}}{\longrightarrow} Y_{q} \stackrel{d_{q}}{\longrightarrow} \ldots \rightarrow Y_{2} \stackrel{d_{2}}{\longrightarrow} Y_{1} \stackrel{d_{1}}{\longrightarrow} Y_{0} \stackrel{d_{0}}{\longrightarrow} Z G \stackrel{\varepsilon}{\longrightarrow} Z \rightarrow 0
$$

where

$$
\mathrm{Y}_{0} \text { is a free right ZG-module on }\left\{\alpha_{1}^{0}, \ldots, \alpha_{m_{0}}^{0}\right\} \text {, }
$$


$Y_{1}$ is a free right ZG-module on $\left\{\alpha_{1}^{1}, \ldots, \alpha_{m_{1}}^{1}\right\}$,

$Y_{q}$ is a free right ZG-module on $\left\{\alpha_{1}^{q}, \ldots, \alpha_{m_{q}}^{q}\right\}$.

For $q \geq 2$. The ZG-homomorphisms are $\varepsilon, d_{0}, d_{l}$ as in Theorem 2.2 and for each

$q \geq 2 \quad d_{q}\left(\alpha_{l}^{q}\right)=\sum_{i=1}^{m_{q-1}} \alpha_{i}^{q-1} \lambda_{i j}^{q}$ where $\left(\lambda_{i j}^{q}\right)=\left(b_{i j}^{q}\right)=\left(B_{q}\right)^{t}$ the transpose of the associate $B_{q}$ of $A_{q}$

\section{REFERENCES}

Akhter, N. and S. Majumdar. 2003. Determination of the homology and the cohomology of NEC group with non-orientable orbit spaces and no reflexions. Bull. Call. Math. Soc. 95(3): 207218.

Anick, D. J. (1986), On the homology on associative Algebra, Trans. Amer. Math. Soc., 296, p. 641-654.

Carbone, Lisa, J. 1996. A Filluation of the chain complex of a rewriting system, DIMACS series in Discrete Mathematics and Theoretical computer Science 25.

Eilenberg, S. and S. MacLane. 1954. On the groups $H(\pi, n)$, I, Ann Math. 58(1953), 55-106, II. Methods of computation, Ann math. 60 (1954), 49-139.

Fox, R.H. 1953. Free Differential Calculus I, Ann. Math. 57(3): 547-560.

Gildenhuys, D. 1976. Generalisaions of Lyndon's theorem on the cohomoloy of one relator groups, Canada. J. Math. 28: 473-480.

Groves, J.R.J. 1989. Rewriting systems and homology groups, Proceedings of the third International conference on the theory of Groups, Canberra, (1456 LNM, Springer-Verlag).

Gruenberg, K.W. 1960. Resolution of relations. J. London Math. Soc. 35: 481-494.

Huebschmann J. 1989. The mod $p$ cohomology rings of metacyclic group. J. Pure Appl. Algebra. 60: 53-103.

Huebschmann, J. 1989. Cohomology of nilpotent groups of class 2. J. Algebra. 126: 400-450

Huebschmann, J. 1989. Perturbation theory and free resolutions for nilpotent groups of class 2. J. Algebra 126: 348-399.

Huebschmann, J. 1993. Cohomological theory of aspherical groups and of small cancellation groups. J. Pure Appl. Algebra 14: 137-143.

Learner, A. 1965. Cohomology groups, Lecturer notes, Queen Mary College, University of London.

Lyndon, R.C. 1950. Cohomology theory of groups with a single defining relation, Ann. Math. 52 (3): 656-665.

MacLane, S. 1963. Leray-Serre theorem. In: Homology, Springer-Verlag, Berlin.

Majumdar, S. 1970. A free resolution for a class of groups. J. Lond. Math. Soc. 2(2): 615-619.

Majumdar, S. 1971. A free resolution for a general class of groups, Tech. report Math. stat. (17), 17, ISI, Calcutta.

Majumdar, S. 1983. Free resolution for certain classes of groups, Proc. Edin. Math. Soc. 26: 173179.

Majumdar, S. 1984. Homology and cohomology of a class of polycyclic groups. Internal report, IC/84/177, ICTP, Trieste, Italy. 
Majumdar, S. 1987. On the homology and cohomology of certain polycyclic groups, Internal report, IC/87/305, ICTP, Trieste, Italy.

Majumdar, S. and N. Akhter. 2002. Determination of the homology and the cohomolgy of the trifoil knot group and the fundamental group of an interesting manifold. J. Bangladesh Math. Soc. 22: 7-14.

Majumdar, S. and N.Akhter. 2008. Determination of the homology and the cohomolgy of a class of infinite groups associated with groups of Fibonacci type. Bull. Call. Math. Soc. 100.

Squire, C.C. 1987. Word problems and a homological finiteness condition for modules. J. Pure Appl. Algebra 40: 201-217.

Swan, R.G. 1969. Group of cohomological dimension one. J. Algebra. 12: 565-610.

Wall, C.T.C. 1961. Resolutions for extensions of groups. Proc. of the Phil. Soc. 57: 251-255.

(Received revised manuscript on 13 June, 2010) 